\title{
A Characterization of DFS Cotree Critical Graphs
}

\author{
Hubert de Fraysseix ${ }^{1}$ and Patrice Ossona de Mendez ${ }^{2}$ \\ 1 UMR 8557, CNRS, Paris, France \\ hf@ehess.fr, http://www.ehess.fr/centres/cams/person/hf/ \\ 2 UMR 8557, CNRS, Paris, France \\ pom@ehess.fr, http://www.ehess.fr/centres/cams/person/pom/
}

\begin{abstract}
We give a characterization of DFS-cotree critical graphs which is central to the linear time Kuratowski finding algorithm implemented in PIGALE1.
\end{abstract}

\section{Introduction}

The present paper is a part of the theoretical study underlying a linear time algorithm finding a Kuratowski subdivision in a non planar graph ([1]; see also [7. for another algorithm). It relies on the concept of DFS cotree critical graph, which is an efficient sub-product of DFS-based planarity testing algorithms (such as 4 and [3]). Roughly speaking, a DFS-cotree critical graph is a simple graph of minimum degree 3 having a DFS tree, such that any non-tree (i.e. cotree) edge is critical, in the sense that its deletion would lead to a planar graph. A first study of DFS-cotree critical appeared in [2], in which it is proved that a DFS-cotree critical graph either is isomorphic to $K_{5}$ or includes a subdivision of $K_{3,3}$ and no subdivision of $K_{5}$. We give here a full characterization of DFS-cotree critical graphs: a simple graph is DFS-cotree critical if and only if it is either $K_{5}$, or a Möbius pseudo-ladder having a simple path including all the non critical edges (see Figure 1).

\section{Preliminary Definitions}

A Möbius pseudo-ladder is a natural extension of Möbius ladders allowing triangles. This may be formalized by the following definition.

Definition 1. A Möbius pseudo-ladder is a non planar simple graph, which is the union of a polygon $\left(v_{1}, \ldots, v_{n}\right)$ and chords (called bars) such that any two non adjacent bars are interlaced (recall two non adjacent edges $\left\{v_{i}, v_{j}\right\}$ and $\left\{v_{k}, v_{l}\right\}$ are interlaced if, in circular order, one finds exactly one of $\left\{v_{k}, v_{l}\right\}$ between $v_{i}$ and $v_{j}$ ).

\footnotetext{
${ }^{1}$ PIGALE, which stands for "Public Implementation of a Graph Algorithm Library and Editor", is freely available at ftp://pr.cams.ehess.fr/pub/pigale.tar.gz

P. Mutzel, M. Jünger, and S. Leipert (Eds.): GD 2001, LNCS 2265, pp. 84-95, 2002 (C) Springer-Verlag Berlin Heidelberg 2002
} 

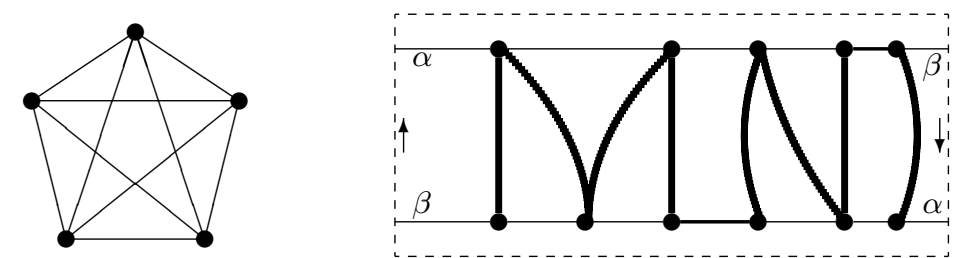

Fig. 1. The DFS-cotree critical graphs are either $K_{5}$ or a Möbius pseudo-ladders having all its non critical edges (thickest) included in a single path

This definition means that a Möbius pseudo-ladder may be drawn in the plane as a polygon and internal chords such that any two non adjacent chords cross. In the projective plane, a Möbius pseudo ladder may be drawn without crossing (see Figure 2). Notice that $K_{3,3}$ and $K_{5}$ are both Möbius pseudo-ladders.
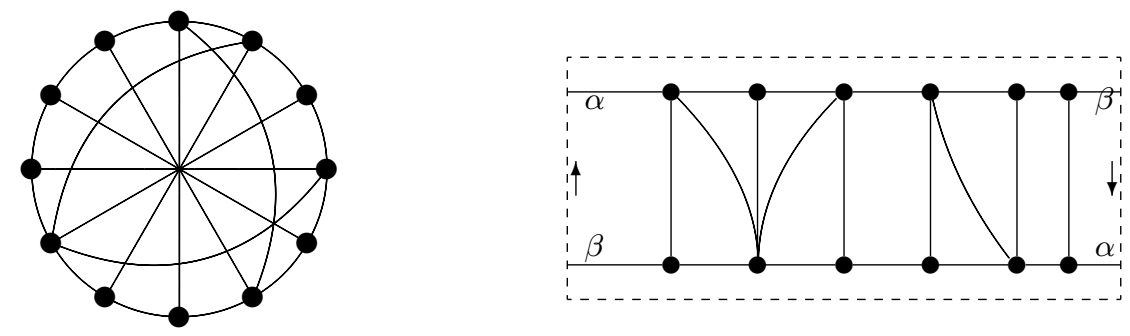

Fig. 2. A Möbius pseudo-ladder on the plane and on the projective plane

Definition 2. Let $G$ be a graph. An edge e $\in E(G)$ is critical if $G-e$ is planar.

Definition 3. An almost planar graph is a graph, such that the set of non critical edges of $G$ is acyclic.

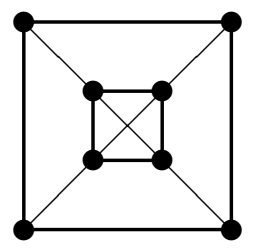

Fig. 3. A non planar graph with bold non-critical edges (notice that the set of noncritical edges is not acyclic and hence the graph is not almost planar) 
The introduction of cotree critical graphs rely on the following reduction/ extension lemma:

Lemma 1. Let $G$ be a graph and let $H$ be the graph obtained from $G$ by recursively deleting all the vertices of degree 1 and contracting all the simple paths into single edges. Then, $G$ is almost planar if and only if $H$ is almost planar.

Proof. First notice that the critical edges of $G$ that remains in $H$ are critical edges of $H$, according to the commutativity of deletion and contraction operations (for $e \in E(H)$, if $G-e$ is planar so is $H-e$ ).

Assume $G$ is almost planar. Then, for any simple path $P$ of $G$, either all the edges of $P$ are critical or they are all non critical. Hence, if $H$ had a cycle of non critical edges, they would define a cycle of non critical edges of $G$.

Conversely, assume $H$ is almost planar. Adding a vertex of degree 1 does not change the status of the other edges and cannot create a cycle of non critical edges. Similarly, subdividing an edge creates two edges with the same status without changing the status of the other edges and hence cannot create a cycle of non critical edges.

Definition 4. A cotree critical graph is a almost planar non-planar graph, with minimum degree 3.

If $G$ is non planar and if $K$ is a Kuratowski subdivision in $G$, it is clear that any critical edge of $G$ belongs to $E(K)$. This justify a special denomination of the vertices and branches of a Kuratowski subdivision:

Definition 5. Let $G$ be a non planar graph and let $K$ be a Kuratowski subdivision of $G$. Then, a vertex is said to be a $K$-vertex (resp. a $K$-subvertex, resp. a $K$-exterior vertex) if it is a vertex of degree at least 3 of $K$ (resp. a subdivision vertex of $K$, resp. a vertex not in $K)$. A $K$-branch is the subdivided path of $K$ between two $K$-vertices. A $K$-branch is critical if it includes at least one critical edge.

\section{Cotree Critical Graphs}

We shall first recall the following result on cotree critical graphs (expressed here with our terminology):

Theorem 1 (Fraysseix, Rosenstiehl [2]). A cotree critical graph is either a hut or includes a subdivision of $K_{3,3}$ but no subdivision of $K_{5}$.

Lemma 2. Let $G$ be a cotree critical graph and let $K$ be a Kuratowski subdivision of $G$ isomorphic to $K_{3,3}$. Then, there exists in $E(G) \backslash E(K)$ no path between:

- two vertices (K-vertices or $K$-subvertices) of a same $K$-branch of $K$,

- two $K$-subvertices of $K$-adjacent $K$-branches of $K$. 


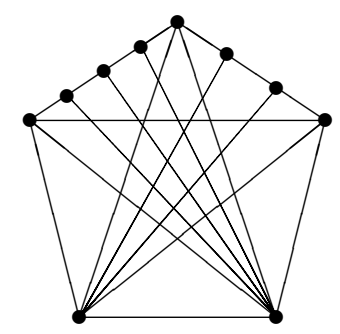

Fig. 4. A hut drawn as a Möbius pseudo-ladder
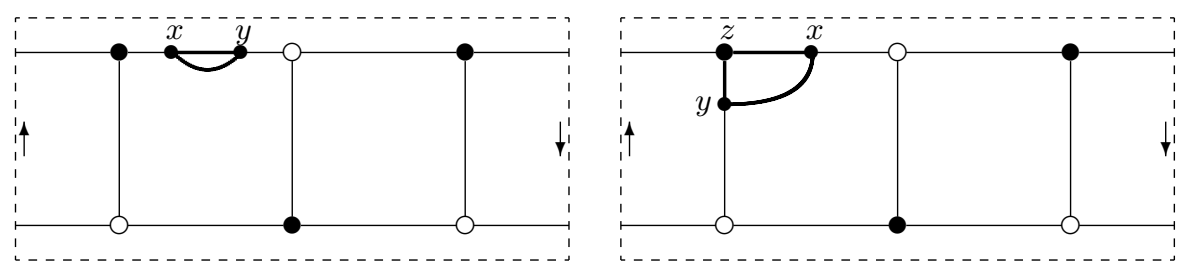

Fig. 5. Forbidden paths in cotree critical graphs (see Lemma 21)

Proof. The two cases are shown Fig 5

If two vertices $x$ and $y$ ( $K$-vertices or $K$-subvertices) of a same $K$-branch of $K$ are joined by a path, both this path and the one linking $a$ and $y$ in $K$ are non critical. Hence $G$ is not cotree critical, a contradiction.

If two $K$-subvertices $x$ and $y$ of $K$-adjacent $K$-branches of $K$ are linked by a path, this path is non critical. Moreover, if $z$ is the $K$-vertex adjacent to the branches including $x$ and $y$, both paths from $z$ to $x$ and $x$ to $y$ are non critical. Hence, $G$ includes a non critical cycle, a contradiction.

Lemma 3. Every cotree critical graph is 3-connected.

Proof. Let $G$ be a cotree critical graph. Assume $G$ has a cut-vertex $v$. Let $H_{1}, H_{2}$ be two induced subgraphs of $G$ having $v$ as their attachment vertex and such that $H_{1}$ is non planar. As $G$ has no degree 1 vertex, $H_{2}$ includes a cycle. All the edges of this cycle are non critical, a contradiction. Hence, $G$ is 2-connected.

Assume $G$ has an articulation pair $\{v, w\}$ such that there exists at least two graphs $H_{1}, H_{2}$ different from a path having $v, w$ as attachment vertices. As $G$ is non planar, we may choose $H_{1}$ in such a way that $H_{1}+\{v, w\}$ is a non planar graph (see [6], for instance). As there exists in $H_{2}$ two disjoints paths from $v$ to $w$, no edge of these paths may be critical and $H_{2}$ hence include a cycle of non critical edges, a contradiction.

Lemma 4. Let $G$ be a cotree critical graph and let $K$ be a Kuratowski subdivision of $G$. Then, $G$ has no $K$-exterior vertices, that is: $V(G)=V(K)$. 

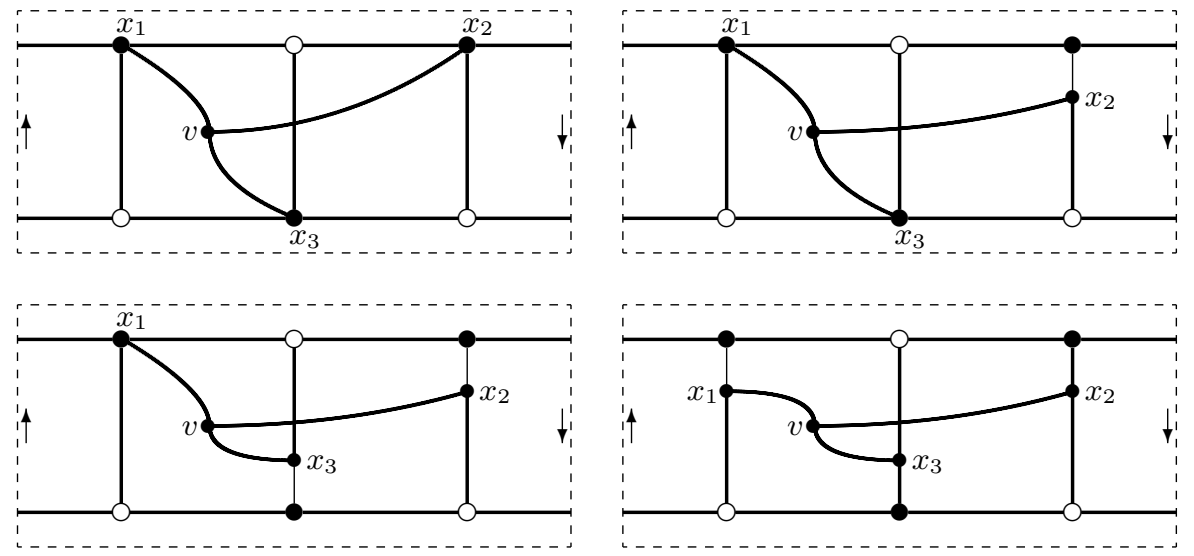

Fig. 6. A cotree critical graphs has no $K$-exterior vertex (see Lemma 4 )

Proof. Assume $V(G) \backslash V(K)$ is non empty and let $v$ be a vertex of $G$ not in $K$. According to Lemma 3, $G$ is 3-connected. Hence, there exists 3 disjoint paths $P_{1}, P_{2}, P_{3}$ from $v$ to $K$. As $K+P_{1}+P_{2}+P_{3}$ is non planar partial subgraph of $G$ free of vertices of degree 1 , it is a subdivision of a 3-connected graph, according to Lemma 1 and Lemma 3 . Thus, the vertices of attachment $x_{1}, x_{2}, x_{3}$ of $P_{1}, P_{2}, P_{3}$ in $K$ are all different. According to Lemma 2, no path in $E(G) \backslash E(H)$ may link $K$-vertices with different colors. Thus, we may assume no white $K$-vertex belong to $\left\{x_{1}, x_{2}, x_{3}\right\}$ and four cases may occur as shown Fig 6. All the four cases show a cycle of non critical edges, a contradiction.

Corollary 1. If $G$ is cotree critical, no non critical $K$-branch may be subdivided, that is: every non critical $K$-branch is reduced to an edge.

Proof. If a branch of $K$ is non critical, there exists a $K_{3,3}$ subdivision avoiding it. Hence, the branch is reduced to an edge, according to Lemma 3 .

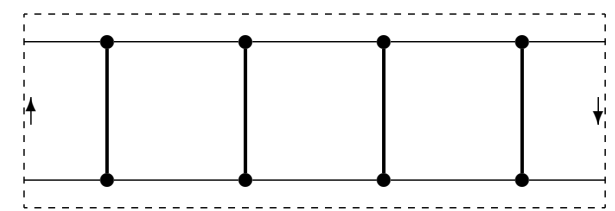

Fig. 7. The 4-bars Möbius ladder $M_{4}$ (all bars are non critical) 
Let $G$ be a cotree-critical graph obtained by adding an edge linking two subdivision vertices of non adjacent edges of a $K_{3,3}$. This graph is unique up to isomorphism and is the Möbius ladder with 4 non critical bars shown Figure 7.

The same way we have introduced $K$-vertices, $K$-subvertices and $K$-branches relative to a Kuratowski subdivision, we define $M$-vertices, $M$-subvertices and $M$-branches relative to a Möbius ladder subdivision.

Lemma 5. Let $G$ be a cotree critical graph and $\{x, y, z\},\left\{x^{\prime}, y^{\prime}, z^{\prime}\right\}$ the $K$ vertices of a $K_{3,3}$ subdivision in $G$.

If $\left[x, z^{\prime}\right]$ or $\left[x^{\prime}, z\right]$ is a critical $K$-branch, then all the edges from $] x, z[$ to ]$x^{\prime}, z^{\prime}\left[\right.$ and the $K$-branch $\left[y, y^{\prime}\right]$ are pairwise interlaced, with respect to the cycle $\left(x, y^{\prime}, z, x^{\prime}, y, z^{\prime}\right)$.

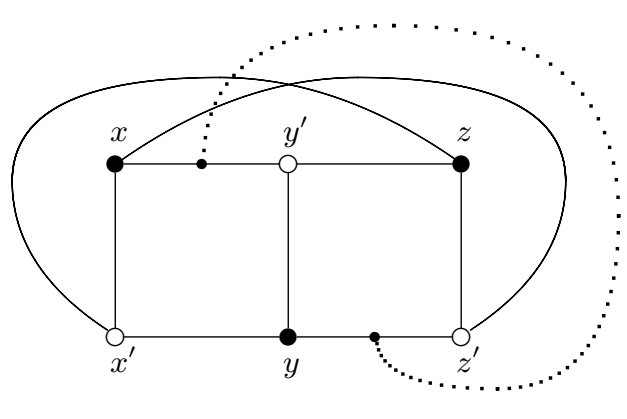

Fig. 8. No edges is allowed from $] x, z[$ to $] x^{\prime}, z^{\prime}[$ by the "outside" (see Lemma [5)

Proof. The union of the $K_{3,3}$ subdivision and all the edges of $G$ incident to a vertex in ] $x, z$ [ and a vertex in ] $x^{\prime}, z^{\prime}$ [ becomes uniquely embeddable in the plane when removing of the $K$-branches $\left[x, z^{\prime}\right]$ or $\left[x^{\prime}, z\right]$. Figure 8 displays the outline of a normal drawing of $G$ is the plane which becomes plane when removing any of the $K$-branch $\left[x, z^{\prime}\right]$ or $\left[x^{\prime}, z\right]$. By planarity, given that an edge from $] x, z[$ to ]$x^{\prime}, z^{\prime}$, if drawn outside, crosses both $\left[x, z^{\prime}\right]$ and $\left[x^{\prime}, z\right]$, all the edges from $] x, z[$ to ]$x^{\prime}, z^{\prime}\left[\right.$ and the $K$-branch $\left[y, y^{\prime}\right]$ are drawn inside the cycle $\left(x, y^{\prime}, z, z^{\prime}, y, x^{\prime}\right)$ without crossing and thus are interlaced with respect to the cycle $\left(x, y^{\prime}, z, x^{\prime}, y, z^{\prime}\right)$. The result follows.

Lemma 6. If $G$ is a cotree critical graph having a subdivision of Möbius ladder $M$ with 4 bars as a subgraph, then it is the union of a polygon $\gamma$ and non-critical chords. Moreover the 4 bars $b_{1}, b_{2}, b_{3}, b_{4}$ of $M$ are chords and any other chord is interlaced with all of $b_{1}, b_{2}, b_{3}, b_{4}$ with respect to $\gamma$.

Proof. Let $G$ be a cotree critical graph having a subdivision of Möbius $M$ ladder with 4 bars $b_{1}, b_{2}, b_{3}, b_{4}$ as a subgraph. First notice that all the bars of the Möbius ladder are non critical and that, according to Corollary 1, they are hence reduced to edges. According to Lemma 4, $M$ covers all the vertices of $G$ as it includes a 
$K_{3,3}$ and hence the polygon $\gamma$ of the ladder is Hamiltonian. Thus, the remaining edges of $G$ are non critical chords of $\gamma$.

Let $e$ be a chord different from $b_{1}, b_{2}, b_{3}, b_{4}$.

- Assume $e$ is adjacent to none of $b_{1}, b_{2}, b_{3}, b_{4}$.

Then it can't be interlaced with less than 3 bars, according to Lemma 2 considering the $K_{3,3}$ induced by at least two non interlaced bars. It can't also be interlaced with 3 bars, according to Lemma 5, considering the $K_{3,3}$ induced by the 2 interlaced bars (as $\left\{x, x^{\prime}\right\},\left\{z, z^{\prime}\right\}$ ) and one non interlaced bar $\left(\right.$ as $\left.\left\{y, y^{\prime}\right\}\right)$.

- Assume $e$ is adjacent to $b_{1}$ only.

Then it can't be interlaced with less than 4 bars, according to Lemma 2 considering the $K_{3,3}$ induced by $b_{1}$ and two non interlaced bars.

- Assume $e$ is adjacent to $b_{1}$ and another bar.

Then it can't be interlaced with less than 4 bars according to Lemma 2 again.

Theorem 2. If $G$ is a cotree critical graph having a subdivision of Möbius ladder $M$ with 4 bars as a subgraph, then it is a Möbius pseudo-ladder whose polygon $\gamma$ is the set of the critical edges of $G$.

Proof. According to Lemma 6 is the union of a polygon $\gamma$ and chords including the 4 bars of $M$. In order to prove that $G$ is a Möbius pseudo-ladder, it is sufficient to prove that any two non adjacent chords are interlaced with respect to that cycle. We choose to label the 4 bars $b_{1}, b_{2}, b_{3}, b_{4}$ of $M$ according to an arbitrary traversal orientation of $\gamma$. According to Lemma 6 , any chord $e$ is interlaced with all of $b_{1}, b_{2}, b_{3}, b_{4}$ and hence its endpoints are traversed between these of two consecutive bars $b_{\alpha(e)}, b_{\beta(e)}$ (with $\left.\beta(e) \equiv \alpha(e)+1 \quad(\bmod 4)\right)$, what well defines the $\alpha$ and $\beta$ functions from the chords different from $b_{1}, b_{2}, b_{3}, b_{4}$ to $\{1,2,3,4\}$.

As all the bars are interlaced pairwise and as any chord is interlaced with all of them, we only have to consider two non adjacent chords $e, f$ not in $\left\{b_{1}, b_{2}, b_{3}, b_{4}\right\}$.

- Assume $\alpha(e)$ is different from $\alpha(f)$.

Then, the edges $e$ and $f$ are interlaced, as $f$ is interlaced with both $b_{\alpha(e)}$ and $b_{\beta(e)}$.

- Assume $\alpha(e)$ is equal to $\alpha(f)$.

Let $b_{i}, b_{j}$ be the bars such that $j \equiv \beta(e)+1 \equiv \alpha(e)+2 \equiv i+3 \quad(\bmod 4)$. Then, considering the $K_{3,3}$ induced by $\gamma$ and the bars $b_{i}, b_{\alpha(e)}, b_{j}$, it follows from Lemma 6 that $e$ and $f$ are interlaced.

\section{DFS Cotree Critical Graphs}

An interesting special case of cotree critical graphs, the DFS cotree critical graphs, arise when the tree may be obtained using a Depth-First Search, as it happens when computing a cotree critical subgraph using a planarity testing 
algorithm. Then, the structure of the so obtained DFS cotree critical graphs appears to be quite simple and efficient to exhibit a Kuratowski subdivision.

In this section, we first prove that any DFS cotree graph with sufficiently many vertices includes a Möbius ladder with 4 bars as a subgraph and hence are Möbius pseudo-ladders, according to Theorem 2 We then prove that these Möbius pseudo-ladders may be fully characterized.

Before proceeding, we shall recall some basic properties of DFS trees.

Definition 6. A DFS tree of a connected graph $G$ rooted at $v_{0} \in V(G)$ may be recursively defined as follows: If $G$ has no edges, the empty set if a DFS tree of $G$. Otherwise, let $G_{1}, \ldots, G_{k}$ the the connected components of $G-v_{0}$, then a DFS tree of $G$ is the union of the DFS trees $Y_{1}, \ldots, Y_{k}$ of $G_{1}, \ldots, G_{k}$ rooted at $v_{1}, \ldots, v_{k}$ (where $v_{1}, \ldots, v_{k}$ are neighbors of $v_{0}$ in $G$ ), and the edges $\left\{v_{0}, v_{1}\right\}, \ldots,\left\{v_{0}, v_{k}\right\}$.

Definition 7. A DFS cotree-critical graph $G$ is a cotree critical graph, which non-critical edge set may be extended into a DFS-tree of $G$.

Lemma 7. If $G$ is $k$-connected $(k \geq 1)$ and $Y$ is a DFS tree of $G$ rooted at $v_{0}$, then there exists a unique chain from $v_{0}$ in $Y$ of length $k-1$.

Proof. According to Definition [7 the lemma is satisfied for $k=1$. Assume the lemma is true for $k \geq 1$, and let $v_{0}$ be a vertex of a $k+1$-connected graph $G$. Then, $G-v_{0}$ has a unique connected component $H$ which is $k$-connected. A DFS tree $Y_{G}$ of $G$ will be the union of a DFS tree $Y_{H}$ of $H$ rooted at a neighbor $v_{1}$ of $v_{0}$ and the edge $\left\{v_{0}, v_{1}\right\}$. As there exists, by induction, a unique chain from $v_{1}$ in $Y_{H}$ of length $k-1$, there will exist a unique chain from $v_{0}$ in $Y_{G}$ of length $k$.

Corollary 2. If $G$ is 3-connected and $Y$ is a DFS tree of $G$ rooted at $v_{0}$, then $v_{0}$ has a unique son which also has a unique son.

Lemma 8. Let $G$ be a cotree critical graph and let $K$ be a Kuratowski subdivision of $G$ isomorphic to $K_{3,3}$. Then, two non $K$-adjacent $K$-vertices a, $b$ cannot be adjacent to $K$-subvertices on a same $K$-branch.

Proof. The three possible cases are shown Figure 9, in all cases, a cycle of non critical edges exist.

Lemma 9. Let $G$ be a cotree critical graph and let $K$ be a Kuratowski subdivision of $G$ isomorphic to $K_{3,3}$. If $G$ has two edges interlaced as shown Figure 10 then $G$ is not DFS cotree critical.

Proof. Assume $G$ is cotree critical. By case analysis, one easily checks that any edge of $G$ outside $E(K)$ is either incident to $a$ or $b$. Hence, all the vertices of $G$ incident to at most one non critical edge is adjacent to a vertex incident with at least 3 non critical edges ( $a$ or $b$ ). According to Corollary 2, the non critical edges cannot be extended to a DFS tree of $G$, so $G$ is not DFS cotree critical. 


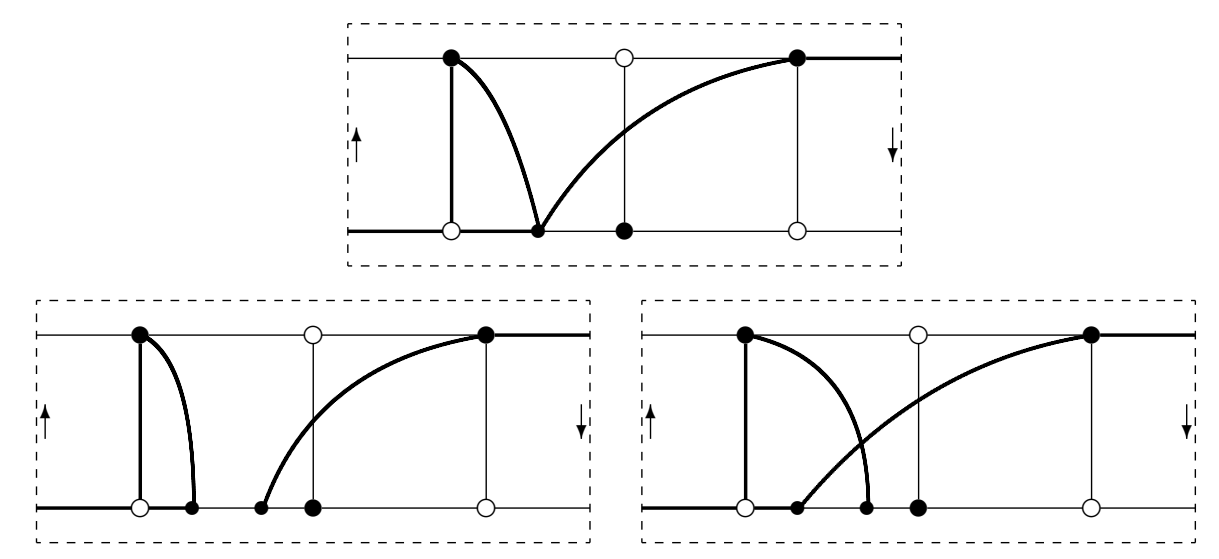

Fig. 9. No two non-adjacent $K$-vertices may be adjacent to $K$-subvertices on the same $K$-branch (see Lemma 8 )

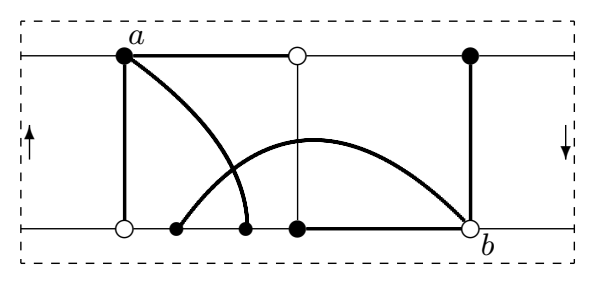

Fig. 10. Case of two adjacent $K$-vertices adjacent to $K$-subvertices on the same $K$ branch by two interlaced edges (see Lemma 9)

Lemma 10. Let $G$ be a DFS-cotree critical graph and let $K$ be a $K_{3,3}$ subdivision in $G$. Then, no two edges in $E(G) \backslash E(K)$ may be incident to a same $K$-vertex.

Proof. Four cases may occur, as shown Figure 11. By a suitable choice of the Kuratowski subdivision, the last two cases are easily reduced to the two first ones.

- Consider the first case.

Assume there exists a $K$-subvertex $v$ between $x$ and $y$. Then, $v$ is not adjacent to a $K$-vertex different from $a$, according to Lemma 8 and Lemma 9. If $v$ were adjacent to another $K$-subvertex $w$, the graph would include a Möbius ladder with 4 bars as a subgraph and, according to Theorem 2 , would be a Möbius pseudo-ladder in which $\{a, y\}$ and $\{v, w\}$ would be non interlaced chords, a contradiction. Thus, $v$ may not be adjacent to a vertex different from $a$ and we shall assume, without loss of generality, that no $K$-subvertex exists between $x$ and $y$. 


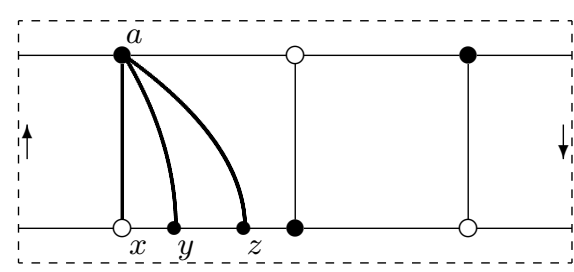

介

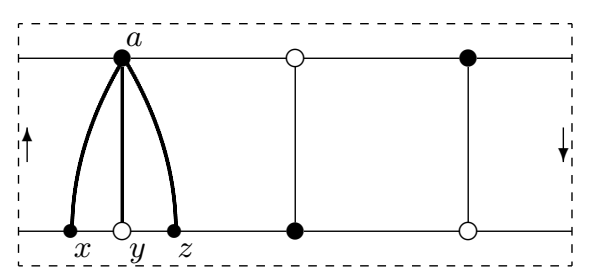

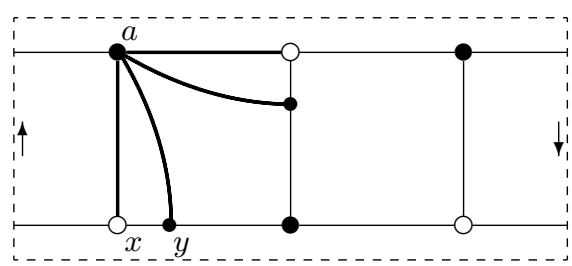

$\Uparrow$

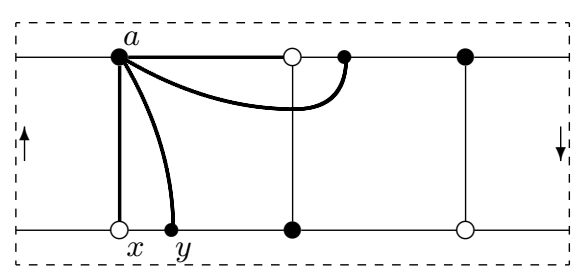

Fig. 11. Cases of Lemma 10

Therefore, if $G$ is DFS-cotree critical, $y$ cannot be a son of $a$, as it would be adjacent (by the left or by the right) to another son of $a$. Hence, vertex $y$ has to be the father of the vertex $a$. As it may not have degree more than one in the DFS tree, it is the root, what contradicts the 3-connexity of $G$, according to Corollary 2 .

- Consider the second case.

We may assume (by symmetry) that the father of $a$ does not belong to the same branch as $y$ and, as previously, that no $K$-subvertices exists between $x$ and $y$. As both $x$ and $y$ are adjacent to $a$ by non critical edges, they are both sons of $a$, the set of non critical edges may not be extended to a DFS tree of $G$.

Lemma 11. If $G$ is DFS-cotree critical includes a subdivision of $K_{3,3}$ and has at least 10 vertices, then $G$ includes a 4-bars Möbius ladder as a subgraph.

Proof. According to Lemma 10, no two edges in $E(G) \backslash E(K)$ may be incident to a same $K$-vertex. If two adjacent $K$-vertices are linked to two $K$-subvertices, then $G$ includes a 4-bars Möbius ladder as a subgraph. Otherwise, if $G$ has at least 10 vertices, there exists an edge linking two $K$-subvertices and $G$ includes a 4-bars Möbius ladder as a subgraph.

Theorem 3 (Fraysseix, Rosenstiehl [2]). A DFS cotree critical graph is either isomorphic to $K_{5}$ or includes a subdivision of $K_{3,3}$ but no subdivision of $K_{5}$.

Theorem 4. Any DFS-cotree critical graph is a Möbius pseudo-ladder. 
Proof. If $G$ is isomorphic to $K_{5}$, the result holds. Otherwise $G$ includes a subdivision of $K_{3,3}$, according to Theorem 3 Then, the result is easily checked for graphs having up to 9 vertices, according to the restrictions given by Lemma 8 and Lemma 10 and, if $G$ as at least 10 vertices, the result is a consequence of Lemma 11 and Theorem 2

Theorem 5. A simple graph is DFS cotree critical if and only if it is a Möbius pseudo ladder which non-critical edges belong to some Hamiltonian path.

Moreover, if $G$ is DFS cotree critical according to a DFS tree $Y$ and $G$ has at least 9 vertices, then $Y$ is a chain and $G$ is the union of a cycle of critical edges and pairwise interlaced non critical chords.

Proof. If all the non critical graphs belong to some simple path, the set of the non critical edges is acyclic and the graph is cotree critical. Furthermore, as we may choose the tree including the non critical edges as the Hamiltonian path, the graph is DFS cotree critical.

Conversely, assume $G$ is DFS cotree critical. The existence of an Hamiltonian including all the non-critical edges is easily checked for graph having up to 9 vertices. Hence, assume $G$ has at least 10 vertices. According to Theorem 5, $G$ is a Möbius pseudo ladder. By a suitable choice of a Kuratowski subdivision of $K_{3,3}$, it follows from Lemma 10 that no vertex of $G$ may be adjacent to more than 2 non critical edges. Let $Y$ be a DFS tree including all the non critical edges. Assume $Y$ has a vertex $v$ of degree at least 3 . Then, one of the cases shown Figure 12 occurs (as $v$ is incident to at most 2 non critical edges) and hence $v$ is adjacent to a terminal $w$ of $T$. The vertex $w$ cannot be the root, according to Corollary 2. Then, among the two neighbors of $w$ in the polygon of the Möbius pseudo ladder, one is not an ancestor of $w$, what contradicts the fact that $Y$ is a DFS tree (as the cotree edges of a DFS tree always join a vertex to one of its ancestors).
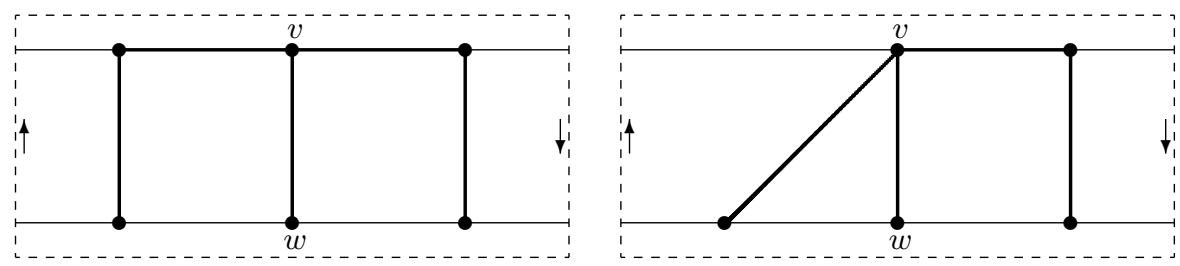

Fig. 12. A vertex of degree at least 3 in the tree is adjacent to a terminal of the tree (see Theorem 5 ) 


\section{References}

1. H. de Fraysseix and P. Ossona de Mendez, An algorithm to find Kuratowski subdivision in DFS cotree critical graphs, Proceedings of the twelfth australasian workshop on combinatorial algorithms (Edy Try Baskoro, ed.), Institut Teknologi Bandung, Indonesia, 2001.

2. H. de Fraysseix and P. Rosenstiehl, A discriminatory theorem of Kuratowski subgraphs, Lecture Notes in Mathematics 1018 (1981), 214-222.

3. _ A characterization of planar graphs by Trémaux orders, Combinatorica 5 (1985), no. 2, 127-135.

4. J.E Hopcroft and R.E. Tarjan, Efficient planarity testing, J. Assoc. Comput. Math. 21 (1974), 549-568.

5. K. Kuratowski, Sur le problème des courbes gauches en topologie, Fund. Math. 15 (1930), 271-293.

6. W.T. Tutte, Graph theory, Encyclopedia of Mathematics and its applications, vol. 21, Addison-Wesley, 1984.

7. S.G. Williamson, Depth-first search and kuratowski subgraphs, Journal of the ACM 31 (1984), no. 4, 681-693. 